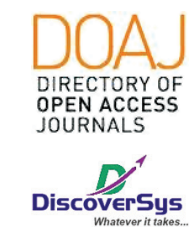

Published by DiscoverSys

\section{Plasmodium falciparum Serine Repeat Antigen 5 (PfSERA5): current vaccine candidate for Plasmodium falciparum malaria}

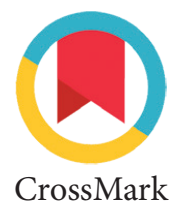

\author{
Ayuti Bulaan, ${ }^{1}$ W. Riski Widya Mulyani, ${ }^{1}$ Agung Nova Mahendra, ${ }^{2 *}$ \\ I Wayan Sumardika ${ }^{2}$
}

\title{
ABSTRACT
}

Background: Most of the malaria cases are caused by Plasmodium falciparum infection. The prevalence of cases and high mortality rates due to malaria should be watched out globally. However, currently, efforts to prevent and treat malaria suffer obstacles due to resistance to insecticides and antimalarial drugs. For these reasons, other preventive measures are needed, such as vaccines. This study aims to review the Plasmodium falciparum Serine Repeat Antigen 5 (PfSERA5) as a potential candidate for Plasmodium falciparum malaria vaccine development.

Methods: 0 the 65 journals reviewed, 51 journals were found to be suitable as references for this paper. The keywords included in selected search engines are "malaria" "PfSERA5", "Plasmodium falciparum", and "malaria vaccine". Search results and studies show that the erythrocytic phase of the vaccine can fight malaria parasites that escape the liver stage while reducing or eliminating clinical symptoms. Data were analyzed and written in a narrative form.

Results: PfSERA5 is an asexual erythrocytic stage antigen that accumulates in the parasitophorous vacuole. The PFSERA5, SE47 and SE36 (modified SE47) domains can induce the formation of antibodies that protect against falciparum malaria infection in vivo and in vitro. This protective mechanism, caused by PFSERA5 (anti-SE47 and anti-SE36) specific antibodies, occurs through inhibition of parasite growth and merozoite lysis. PfSERA5 also does not show antigenic variations and has limited polymorphism, so the probability of resistance can be reduced. Conclusion: Based on this, PfSERA5 has great potential as an effective erythrocytic phase vaccine candidate. However, further studies are needed regarding the toxicological and pharmacological properties of PFSERA5, both in vivo and in clinical settings.

Keywords: Malaria, pfSERA5, Plasmodium falciparum, vaccine

Cite This Article: Bulaan, A., Mulyani, W.R.W., Mahendra, A.N., Sumardika, I.W. 2020. Plasmodium falciparum Serine Repeat Antigen 5 (PfSERA5): current vaccine candidate for Plasmodium falciparum malaria. Intisari Sains Medis 11(1): 320-327. D0I: 10.15562/ism.v11i1.573

\section{INTRODUCTION}

Malaria is one of the public health problems caused by protozoan parasites from the genus Plasmodium. According to WHO, around 212 million cases of malaria occurred globally in 2015, with a relatively high mortality rate of $62 \% .^{1}$ In Indonesia, malaria was ranked second in infectious diseases after pulmonary TB, with the most prevalent malaria parasite species being Plasmodium falciparum (86.4\%). ${ }^{2}$ In 2013, Riskesdas reported the highest incidence and prevalence of malaria in Indonesia occurred in five provinces of Papua, East Nusa Tenggara, West Papua, Central Sulawesi, and Maluku. This high prevalence of cases and mortality rates make malaria an infectious disease that should be watched out globally so that every country has a commission to eliminate this infectious disease. ${ }^{3}$ Vector control is an essential component in preventing malaria. Such control is successful in reducing or disrupting malaria transmission when coverage is high. Two types of malaria vector control methods recommended by WHO are long-lasting insecticidal nets (LLINs) and indoor residual spraying (IRS). ${ }^{4}$ Both are carried out in Indonesia following malaria elimination procedures. Unfortunately, in recent years, the resistance of malaria vectors to insecticides used in these two methods has increasingly emerged in various countries. This would significantly hamper vector control, especially in endemic areas vulnerable to resistance. ${ }^{5}$

Meanwhile, for malaria treatment, the Annual Parasite Incidence (API) indicator shows that currently malaria positive cases proven through blood tests must be treated using an artemisinin or Artemisinin-based Combination Therapies (ACT) based combination. ${ }^{6}$ ACT is more effective for malaria treatment than other anti-malarial drugs because malaria parasites have experienced resistance to almost all anti-malarial drugs. Treatment with ACT has significantly contributed to the treatment of malaria over the past few years. ${ }^{7}$ However, in 2006, resistance to ACT also began to be detected in various regions of the world and is currently widespread in Southeast Asia. ${ }^{8,9}$ Extensive 
artemisinin resistance is predicted to cause more than 116,000 deaths annually. ${ }^{10}$ Research shows that anti-malarial resistance in Plasmodium falciparum occurs due to genetic factors, especially mutations in parasites. ${ }^{11}$ Resistance events that happen in both malaria vector resistance to insecticides and Plasmodium falciparum resistance to anti-malarial drugs have inhibited both the prevention of transmission and treatment of positive malaria cases. Therefore, now another more effective prevention method is needed, namely the vaccine. An effective malaria vaccine will be a valuable finding for controlling, eliminating and limiting malaria. RTS, S, a pre-erythrocytic Plasmodium falciparum malaria vaccine is known to have the efficacy of up to $53 \% .{ }^{12}$ In this context; the pre-erythrocytic vaccine presents an alternative by interfering with parasite transmission. However, RTS, S has not been able to produce sterile immunity to fight malaria.

Although this vaccine immunization will induce antibodies that correlate with protection against Plasmodium falciparum infection, there is no evidence that this vaccine can block the malaria parasite in the liver stage and prevent clinical symptoms. Unlike the pre-erythrocytic phase vaccines, erythrocytic phase vaccines will not only fight parasites that have escaped the liver stage but also reduce or eliminate clinical symptoms. In addition, it may reduce the number of sexual stage gametocytes that will be consumed by subsequent Anopheles mosquitoes when sucking the blood of infected individuals. Among the few malaria proteins targeted by potential erythrocytic phase candidates, advances in the Plasmodium falciparum serine repeat antigen (PfSERA) study have provided promising hope in the innovation of falciparum malaria vaccine. ${ }^{13}$ One member of the multigene SERA family in Plasmodium falciparum is serine repeat antigen 5 (PfSERA5). PfSERA5 is an abundant and dissolved asexual stage antigen from the final trophozoite and schizont which accumulates in the parasitophorous vacuole. This antigen is released in the dissolved form at the time the schizont ruptures and can induce the formation of antibodies that protect from erythrocytic malaria infections in vivo, and can inhibit parasitic growth in vitro. ${ }^{14-16}$ In addition, PfSERA5 also does not show antigenic variation and has limited polymorphism. ${ }^{17}$

Based on these data, PfSERA5 has considerable potential as a candidate for the latest malaria falciparum vaccine RTS. A pre-erythrocytic Plasmodium falciparum malaria vaccine is known to have the efficacy of up to $53 \% .{ }^{12}$ In this context; the pre-erythrocytic vaccine presents an alternative by interfering with parasite transmission. However, RTS, S has not been able to produce sterile immunity to fight malaria. Although this vaccine immunization will induce antibodies that correlate with protection against Plasmodium falciparum infection, there is no evidence that this vaccine can block the malaria parasite in the liver stage and prevent clinical symptoms. Unlike the pre-erythrocytic phase vaccines, erythrocytic phase vaccines will not only fight parasites that have escaped the liver stage but also reduce or eliminate clinical symptoms. It may reduce the number of sexual stage gametocytes that will be consumed by subsequent Anopheles mosquitoes when sucking the blood of infected individuals. Among the few malaria proteins targeted by potential erythrocytic phase candidates, advances in the Plasmodium falciparum serine repeat antigen (PfSERA) study have provided promising hope in the innovation of falciparum malaria vaccine. ${ }^{13}$ One member of the multigene SERA family is Plasmodium falciparum serine repeat antigen 5 (PfSERA5). PfSERA5 is an abundant and dissolved asexual stage antigen from the final trophozoite and schizont which accumulates in the parasitophorous vacuole. This antigen is released in the dissolved form at the time the schizont ruptures and can induce the formation of antibodies that protect from erythrocytic malaria infections in vivo, and can inhibit parasitic growth in vitro. ${ }^{14-16}$ In addition, PfSERA5 also does not show antigenic variation and has limited polymorphism. ${ }^{17}$ Based on those mentioned above, PfSERA5 has considerable potential as a candidate for the latest malaria falciparum vaccine.

\section{METHODS}

The writing method used is a literature review. The literature source consists of relevant journals from the search engine www.pubmed.com, proquest.com and scholar.google.com. The author searches with the keywords of "malaria" "PfSERA5", "Plasmodium falciparum", and "malaria vaccine" in the search engine. The inclusion criteria were all malaria samples caused by Plasmodium falciparum parasites, and references did not exceed the last ten years unless there were no new studies that opposed the contents of these references. Of the 65 journals reviewed, 51 journals were found to be suitable as references for this paper. The information collected is recorded, and analyzed for validity and reliability, interpreted and compiled into a study of scientific literature. All of the data provided from the literature then selected, analyzed, and explained by using a narrative approach in systematic and logic order. 


\section{RESULTS}

\section{PfSERA5 potential as Malaria Falciparum Vaccine candidate}

Vaccines are still being studied intensively because of their great potential in eradicating infectious morbidity and mortality, including malaria. The malaria vaccine can be targeted both in the pre-erythrocytic phase and the erythrocytic asexual phase. ${ }^{18}$ However, vaccines in the pre-erythrocytic phase, such as RTS, S have not been able to produce sterile immunity to fight malaria. Although this vaccine immunization will induce antibodies that correlate with protection against Plasmodium falciparum infection, there is no evidence that this vaccine can block the malaria parasite in the hepatic stage and prevent clinical symptoms. ${ }^{12}$ Unlike the asexual erythrocytic vaccine which is not only able to fight parasites that have escaped the liver stage but also reduce or eliminate clinical symptoms and may reduce the number of sexual stages of gametocytes. ${ }^{13}$

Research on erythrocytic vaccines brings up various antigens as vaccine candidates due to immune reactions in the form of complex antigen-antibody interactions. The potential of vaccine candidates is measured by the importance of the role of the antigen in triggering antibody titers by resulting in an adequate protective immune response. ${ }^{12}$ In the development of the malaria vaccine; there are two main antigens often discussed as vaccine candidates, namely Plasmodium falciparum apical membrane antigen 1 (PfAMA1) and Plasmodium falciparum merozoite surface protein 1 (PfMSP1). ${ }^{19,20}$ But extensive genetic diversity parasites due to the selective pressure is given by the human immune response present a major obstacle to the development of this vaccine candidate. These two antigen classes are known to have high polymorphism profiles so that at any time they can change the nature of antigens that cause parasites to avoid the host immune system. ${ }^{21,22}$ This will increase the likelihood of disease resistance. Polymorphism profile determines parasitic susceptibility. The high profile of polymorphisms in both causes vaccines based on PfAMA1 and PfMSP1 must induce high concentrations of antibodies to provide adequate protective effects. ${ }^{6,7}$

In contrast, through sequence analysis of 445 isolates of the Plasmodium falciparum on the field from nine countries in the world, it is known that PfSERA5 does not show antigenic variation and has limited polymorphism so that the possibility of parasites experiencing resistance to PfSERA5 based vaccines will be slighter. PfSERA5-based vaccine candidates can induce the formation of antibodies that protect from erythrocytic phase malaria infections in vivo and can inhibit parasitic growth in vitro. ${ }^{14-16}$

In addition, high antibody levels in response to $\mathrm{N}$ - terminal domains $47 \mathrm{kDa}$ pfSERA5 (commonly called SE47) causes reduced symptoms of fever or parasitemia. ${ }^{23-25}$ In in vitro conditions, SE47induced antibodies correlate with antiparasitic effects through several mechanisms. When the antibody concentrations are high, inhibition of parasite growth is found to be associated with merozoite agglutination or mediated cell lysis complement of segmented schizont. ${ }^{26,27}$ Whereas in low antibody concentrations, inhibition cellular antibody activity depends on monocyte or monocyte- mediated antibody-dependent cellular inhibition (ADCI) is also demonstrated. ${ }^{28}$ The malaria vaccine candidate antigen from the other pfSERA5, SE36, is based on the SE47 domain. This antigen is constructed by removing the glycerin region in the centre of the domain. ${ }^{23}$ The removal of the poliserin region is intended to improve hydrophilicity and reduce the hydrophobicity of proteins to be more easily purified and suitable for clinical evaluation. Recent clinical trials and phase 1 trials for 365 days showed that, after vaccination with SE36 for two weeks, the protective efficacy of $72 \%$ of symptomatic malaria resulted in Ugandan children aged 6- 20 years. ${ }^{29}$

Animal experiments using nonhuman primates, and phase 1 clinical trials in humans assessed the immunogenicity of the SE36 vaccine. ${ }^{23}$ The SE36/ AHG vaccine (GMP-grade SE36 combined with a lighter auxiliary ingredient, aluminium hydroxyl gel) induces specific antibodies in the model of a squirrel monkey infected with malaria. Infection exposure is carried out with a relatively large number of parasites which are unlikely to occur under normal conditions $\left(1 \times 10^{9}\right.$ erythrocytes/ infected monkeys), showing four to six percent parasitemia in control monkeys three days after infection, whereas SE36/AHG immunized monkeys show less parasitemia one percent on the same day, and five to ten times lower peak parasitemia during the rest of the study. ${ }^{23}$ The challenge in anti-SE36 antibody production because of this infection was not observed in the control group. This shows the success of the malaria vaccine from the clinical formulation of the SE36 protein, as well as the ability of SE36/AHG to provide an immune response that can be driven by natural infections. Further confirmation is carried out using chimpanzee immunization experiments. Antibody levels are monitored for two years. Two doses of $450 \mathrm{~g}$ and $50 \mathrm{~g}$, and three doses of $10 \mathrm{~g}$ produced an anti SE36 titer which averaged more than 40 weeks. ${ }^{23}$

The phase 1a clinical trial observed showed treatment with a dose of $50 \mu \mathrm{g} \mathrm{SE36/AHG} \mathrm{and} \mathrm{treat-}$ ment with a dose of $100 \mu \mathrm{g}$ SE36/AHG and placebo 
control. The level of safety and immunogenicity of SE36/AHG was tested in 30 healthy adults and ten placebo controls. Three times subcutaneous administration at a dose of 50 and $100 \mu \mathrm{g}$ SE36/AHG can be well-tolerated, without severe side effects. Three times the dose of $50 \mu \mathrm{g}$ did not produce a high antibody response rate, but twice the dose of $100 \mu \mathrm{g}$ was able to produce it. Nevertheless, treatment with both doses capable of producing seroconversion of $100 \%$. Current research results for SE36/AHG provide initial clinical validation for future trials and strategies for further vaccine development. ${ }^{23}$

\section{PfSERA5 role in the pathogenesis of malaria} falciparum

The pathogenesis of falciparum malaria (Figure 2) begins when a mosquito vector containing Plasmodium falciparum sucks human blood. The pre-erythrocytic cycle occurs when sporozoites in the mosquitors salivary gland are injected into the blood and then migrate to the liver to infect hepatocytes to form merozoites. ${ }^{30}$ In the liver, sporozoites are enlarged because duplication of the nucleus and other organs develops a schizont which then undergoes cytokinesis into thousands of identical cells called merozoites. Furthermore, merozoites will come out of the liver cells because they rupture and enter the bloodstream. The erythrocytic cycle begins when merozoites invade red blood cells using their complex complexes and develop into early trophozoites (immature trophozoites). ${ }^{31,32}$ Immature trophozoites can enter two different pathways, which can develop into mature trophozoites to induce other red blood cells or can also develop into gametocytes. ${ }^{13}$ Mature trophozoites will experience schizogony (asexual splitting) in human erythrocytes to form schizonts and subsequently cause rupture of red blood cells, which then secrete new merozoites. This merozoite can infect other red blood cells. ${ }^{33}$

The erythrocytic phase is the phase of a breakdown in red blood cells and at the same time, the period of the emergence of clinical symptoms of malaria in Plasmodium falciparum infection. An essential protein mediates the rupture of red blood cells from Plasmodium falciparum at the time of the final trophozoite and schizont. These proteins are part of the multigene family called SERA5 (Serine Repeat Antigen 5). ${ }^{34}$ Plasmodium falciparum serine repeat antigen 5 (PfSERA5) is a protein that was first identified as trophozoite and schizont phase proteins. These proteins are soluble, exported, and are abundantly expressed in parasitophorous vacuoles and on the surface of merozoites. This antigen is released in dissolved form when the schizont ruptures. ${ }^{13}$ The most active
PfSERA multigene family transcribed at trophozoite and schizont stages with the most significant percentage of transcription is owned by PfSERA5, which is about $0.5-1.5 \%$ of the total mRNA at the schizont stage. ${ }^{35}$ PfSERA5 is very important for the survival of parasites. ${ }^{36,37}$ Together with at least another essential PfSERA multigene family member (SERA6), SERA5 is believed to mediate the discharge of parasites from infected host cells through cellular processing substrates in cleavage by Plasmodium falciparum subtilisin-like protease 1 (PfSUB1). ${ }^{13,38}$ The mechanism for mediating the removal of parasites by PfSERA5 begins after merozoites invade erythrocytes. Then at the end of the trophozoite stage entering the initial phase of schizont, PfSERA5 accumulates in vacuole parasitophorous as a full fragment (120 kDa protein) (Figure 1).

The $120 \mathrm{kDa}$ PfSERA protein is processed into domains 47 and 73 . Domain 73 will split into domains 56 and $18 .{ }^{13}$ It is in this division of cellular substrates that the role of PfSUB1 is much needed.38 Furthermore, domain 56 will be split into 50 domains and $6 \mathrm{kDa}$ domains. While 50 and $6 \mathrm{kDa}$ domains are secreted, the $50 \mathrm{kDa}$ domain will carry out a proteolytic activity which is believed to be the cause of schizont rupture. Domains 47 and $18 \mathrm{kDa}$ will be covalently connected by disulfide bonds and reside on the surface of the merozoite. ${ }^{39,40}$ Eventually the new merozoites will invade other red blood cells and the cycle will continue. PfSERA5 plays a vital role in the survival of parasites through the mechanism described earlier. PfSERA5 can induce the formation of antibodies that protect against malaria infection in the erythrocytic phase in vivo and inhibit parasitic growth in vitro (Figure 1 and 2). ${ }^{14,15}$ In addition, PfSERA5 also does not show antigenic variations and has limited polymorphism. The profile of the polymorphism of an antigen will determine the parasitic virulence in the host and the specificity of the host immune response to the parasite. ${ }^{39}$

\section{PfSERA5 (Anti-SE47 and Anti-SE36) Specific IgG Protection Mechanism against Plasmodium falciparum}

In the in vitro conditions, some studies have shown that specific IgG PfSERA5 (anti-SE47 and SE36) correlated with antiparasitic effect through two main mechanisms, namely induced lysis of merozoites and inhibition of the growth of the parasite Plasmodium falciparum. When IgG concentration is high, inhibition of parasite growth is found to be associated with merozoite agglutination and merozoite lysis is mediated by the membrane-attack complex (MAC). ${ }^{26,27}$ Whereas at low antibody 


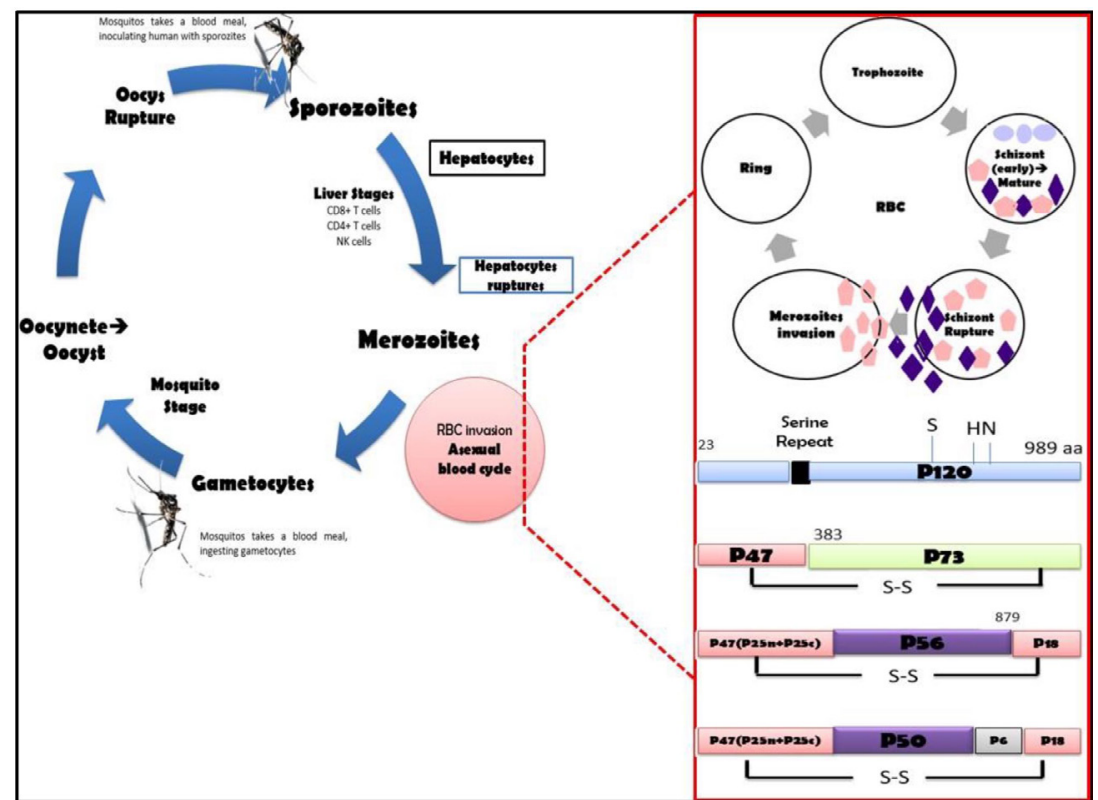

Figure 1 Plasmodium falciparum Life Cycle and PfSERA5 Domain Localization $^{40}$

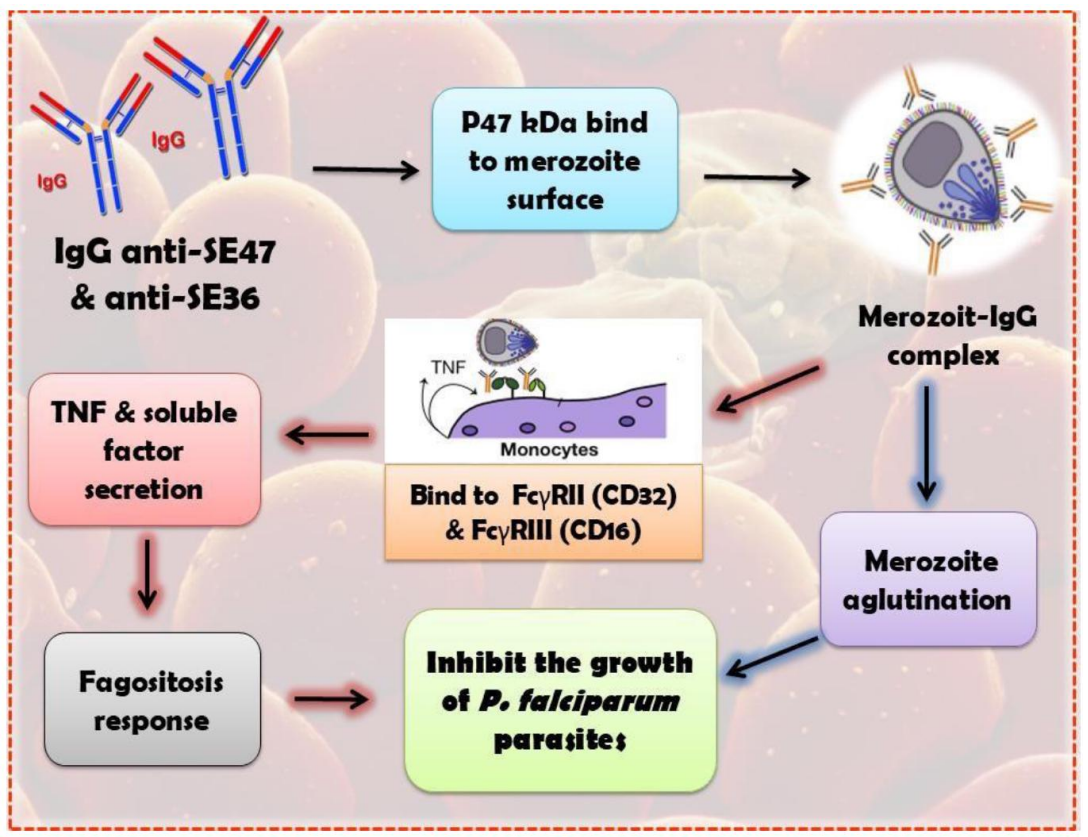

Figure 2 PfSERA5-specific IgG protection mechanism (anti-SE47 and anti-SE36) in inhibiting parasite growth ${ }^{26,28,45}$

concentrations, cellular monocyte-dependent antibody-dependent AD (ADCI) inhibitory cellular activity is also shown which is also capable of inhibiting parasite growth. ${ }^{28}$

PfSERA5 specific IgG protection mechanism (anti-SE47 and anti-SE36) in inhibiting $P$. falciparum parasite growth

Several studies have shown that antibodies induced by pfSERA 5 can inhibit the growth of parasites. ${ }^{41-43}$ Vaccination of rats or goats with recombinant
$\mathrm{N}$-terminal SERA is known to stimulate parasitic inhibiting antibodies. ${ }^{27,42,44}$ Inhibition of parasite growth by acquired immunity naturally in response to the N-terminal pfSERA5 domain, indicating a direct involvement of protein antibodies. ${ }^{35}$ Furthermore, serum in adults in Uganda with high anti-SE47 antibody titers correlates with inhibition of parasite growth. This shows the potential for PfSERA5 specific IgG protection in inhibiting parasite growth (Figure 3).

The earliest IgG response to SE47 or SE36 showed an antibody response that was significantly skewed to IgG3 ${ }^{23,24}$ When induced, anti-SE47 and anti-SE36 IgG will bind merozoite surface antigens, $\mathrm{p} 47 \mathrm{kDA}$ to form a merozoite-IgG complex. This complex will trigger merozoite agglutination which will cause inhibition of the growth of the Plasmodium falciparum parasite. ${ }^{26}$

Besides, the merozoite-IgG complex can also induce monocyte-mediated Antibody-Dependent Cellular Inhibition (ADCI) activity by attaching to the surface of the monocyte. This attachment occurs through binding of merozoite-IgG complexes to Fc $\gamma$ RII (CD32) and Fc $\gamma$ RII (CD16). Then, the monocytes will secrete TNF and other dissolved factors so that it will increase the phagocytic response and inhibit the growth of $P$. falciparum parasites. ${ }^{26,28,45}$

\section{PfSERA5 (anti-SE47 and anti-SE36) specific IgG protection mechanism in inducing} Merozoite lysis

IgG anti-SE47 and anti-SE36 also allow merozoite lysis (Figure 3). As happened in the mechanism of inhibiting parasite growth, IgG will bind merozoite surface antigens, p47 kDA to form a merozoite-IgG complex. This complex will then cause opsonin $\mathrm{C} 3 \mathrm{~b}$ and $\mathrm{C} 4 \mathrm{~b}$ complement deposition, which will then initiate the lethal membrane-attack complex (MAC). Then, this complex will form pores in the merozoite membrane and allow water and ions to enter merozoite cells. Merozoite cells will swell and eventually undergo lysis. ${ }^{27,45}$

\section{Advantages of Vaccines as Malaria Prevention: PfSERA5 as Candidate for Falciparum Malaria Vaccine}

Vaccines have a significant contribution to improving human health and lifespan. The vaccine is a weakened antigen that has the potential to fight the antigen itself. Research on vaccines is highly developed at this time because it is considered to have great potential in eradicating disease morbidity and mortality. In the case of vaccines, antigenic variation has the potential to be the mechanism behind the emergence of vaccine-resistant strains. However, vaccines have several unique features that 


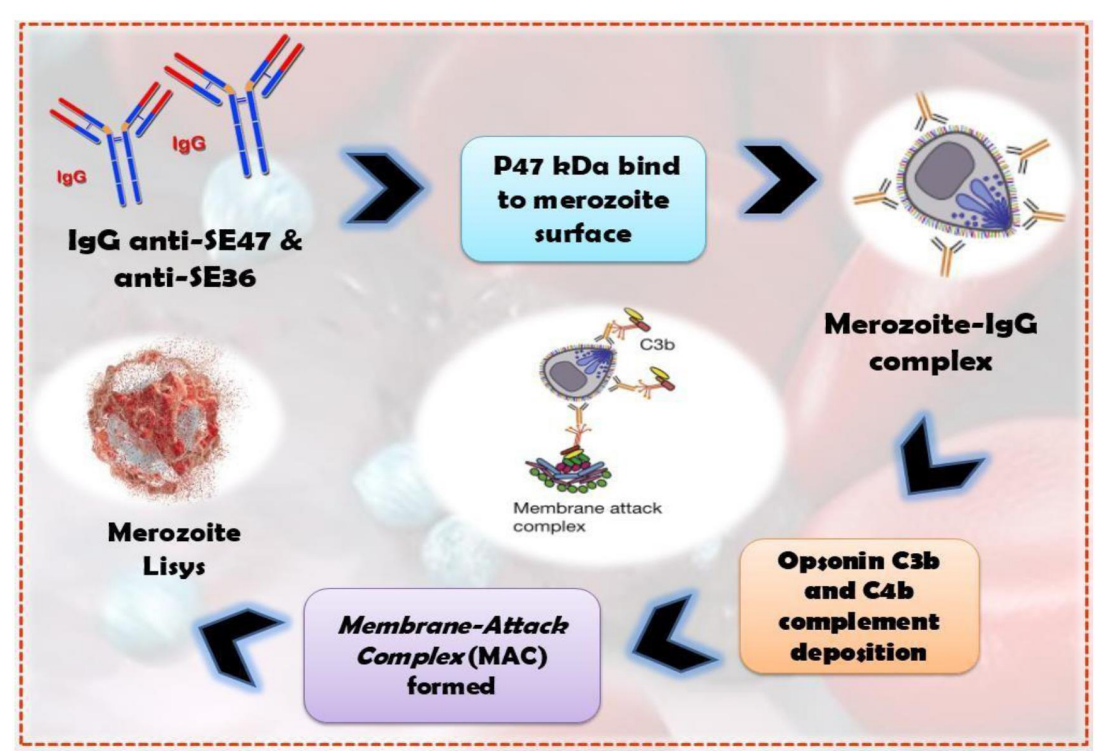

Figure 3 PfSERA 5 (anti-SE47 and anti-SE36) specific IgG protection mechanism in inducing merozoite lysis ${ }^{26,45}$

make them almost resistant to the phenomenon of resistance. Vaccines offer preventive therapy by providing protection that starts from within the human body through induction the immune system that can last for years or even for life. ${ }^{46}$ The ideal falciparum malaria vaccine is a vaccine with antigens that plays an essential role for the survival of parasites and has a broad spectrum of Plasmodium falciparum strains so that resistance will not be easily obtained through mutations. ${ }^{40}$ PfSERA5 is an essential antigen that is very important in the pathogenesis process of malaria parasites. ${ }^{37}$ Based on sequence analysis of $445 \mathrm{P}$. falciparum field isolates from nine countries in the world, it is known that PfSERA5 does not show antigenic variation and has limited polymorphism so that the possibility of parasites experiencing resistance to PfSERA5-based vaccines will be smaller. ${ }^{17}$ High antibody levels in response to the $47 \mathrm{kDa}$ pfSERA5 $\mathrm{N}$-terminal domain (commonly called SE47) cause reduced symptoms of fever or parasitemia. ${ }^{23-25}$ This makes PfSERA5 an up-and-coming vaccine candidate.

The factors that contribute to the parameters of the success of an antigen vaccine are the presence of a response to immunogenicity, stability, availability, high specificity and safety of the vaccine itself. ${ }^{47}$ Just as discussed earlier, PfSERA5 has high immunogenicity, proven in several studies that revealed that PfSERA (anti-SE47 and anti-SE36) could induce IgG antibodies that protect against malaria infection and inhibit parasite growth. Characterization of processing of SERA proteins uses folded proteins that are correctly folded and expressed using a baculovirus expression system. ${ }^{13}$ Anti-SE47 antibodies from recombinant proteins were more resistant when codon-optimized synthetic genes were expressed in E. coli. ${ }^{48}$ This shows the stability and availability of PfSERA5 antigens. The role of PfSERA5, which is very specific in the removal of parasites during schizont rupture makes PfSERA5 a protein that has high specificity and is essential in the pathological process of malaria. PfSERA5 as a vaccine candidate was considered safe based on phase 1a clinical trials which revealed that subcutaneous administration with appropriate SE36/AHG doses could be well tolerated and without severe side effects. ${ }^{23}$ The importance of PfSERA5's specific role in the removal of parasites from erythrocytes that rupture to invade other erythrocytes shows that the development of the SERA recombinant protein-based vaccine is a rational therapeutic modality in efforts to eradicate malaria. Giving vaccines to humans is expected to break the cycle of malaria that will continue in mosquitoes so that the spread of sporozoites and other human infections can be prevented. ${ }^{49}$

\section{Limitations of PfSERA5 as Falciparum Malaria Vaccine Candidate}

In a Ugandan study reported local side effects such as induration, tenderness, swelling, erythema and redness appeared in PfSERA5-based vaccines. However, these symptoms are symptoms that are comparable to other vaccines. ${ }^{50}$ Previously also reported exposure to low $\mathrm{Ca} 2+$ concentrations due to intracellular chelation in schizont, blocking PfSUB1 secretion from exonemes to vacuole parasitophorous and thus inhibiting PfSERA5 processing in the release of merozoites. ${ }^{51}$ Then, signals that regulate temporal activation of protease secretion at the maturation of merozoites in intra-erythrocytic schizonts are still unclear, so commitment from the researchers is needed to investigate those related signalling mechanisms further. ${ }^{51}$ Also, research on the PfSERA5 malaria vaccine, which is currently in the clinical phase of $1 \mathrm{~b}$ and is expected to continue to grow. There is also a need for substantial funding commitments in research into the next phase so that this considerable amount of funding is also one of the problems in conducting further research. ${ }^{13}$ By overcoming existing limitations, the potential of PfSERA5 as a vaccine candidate will be more substantial and its effectiveness will increasingly meet the criteria as one of the preventive therapies in eradicating malaria.

\section{CONCLUSIONS}

After conducting various studies to phase $1 \mathrm{~b}$ clinical trials, PfSERA5 antigens increasingly showed very promising potential as a falciparum malaria vaccine. The high level of immunogenicity, stability, availability, and safety of PfSERA5-based vaccines 
is also an essential factor in evaluating the success of a vaccine candidate. In addition, PfSERA5 does not show antigenic variations and has a limited polymorphism so that the possibility of parasites experiencing resistance to PfSERA5-based vaccines will be slighter. The importance of the role of a vaccine in eradicating the morbidity and mortality of a disease does not completely replace the existing conventional prevention efforts. It should be remembered that holistic prevention is an effort that significantly guarantees the eradication of illnesses given the principle of balance between host, environment, and agent. The authors suggest that a higher level of further research on PfSERA5 antigens is carried out to find out the efficacy and signalling mechanisms of PfSERA5 in the human body. Based on the body of scientific evidence, PfSERA5 can be considered as a promising novel candidate of a falciparum malaria vaccine in the future.

\section{CONFLICT OF INTEREST}

There is no competing interest regarding manuscript.

\section{FUNDING}

None.

\section{AUTHOR CONTRIBUTION}

All of authors are equally contributed to the study from the conceptual framework, data gathering, until analyze and synthesis the literature review through narrative approach.

\section{REFERENCES}

1. Moss WJ, Dorsey G, Mueller I, Laufer MK, Krogstat DJ, Vinetz JM, et al. Malaria Epidemiology and Control Within the International Centers of Excellence for Malaria Research. Am J Trop Med Hyg. 2015;93(3 Suppl):5-15.

2. Sitohang V, Sariwati E, Fajariyani SB, Hwang D, Kurnia B, Hapsari RK, et al. Malaria elimination in Indonesia: halfway there. Lancet Glob Health. 2018;6(6):e604-e606.

3. Hanandita W, Tampubolon G. Geography and social distribution of malaria in Indonesian Papua: a cross-sectional study. Int J Health Geogr. 2016;15:13.

4. Raghavendra K, Barik TK, Reddy BP, Sharma P, Dash AP. Malaria vector control: from past to future. Parasitol Res. 2011;108(4):757-779.

5. Alout H, Labbé P, Chandre F, Cohuet A. Malaria Vector Control Still Matters despite Insecticide Resistance. Trends Parasitol. 2017;33(8):610-618.

6. Davis TM, Karunajeewa HA, Ilett KF. Artemisinin-based combination therapies for uncomplicated malaria. Med J Aust. 2005;182(4):181-185

7. Eastman RT, Fidock DA. Artemisinin-based combination therapies: a vital tool in efforts to eliminate malaria. Nat Rev Microbiol. 2009;7(12):864-874.
8. Nsanzabana C. Resistance to Artemisinin Combination Therapies (ACTs): Do Not Forget the Partner Drug!. Trop Med Infect Dis. 2019;4(1):26.

9. Duru V, Khim N, Leang R, Kim S, Domergue A, Kloeung N, et al. Plasmodium falciparum dihydroartemisinin-piperaquine failures in Cambodia are associated with mutant K13 parasites presenting high survival rates in novel piperaquine in vitro assays: retrospective and prospective investigations. BMC Med. 2015;13:305.

10. Lubell Y, Dondorp A, Guérin PJ, Drake T, Meek S, Ashley J, et al. Artemisinin resistance--modelling the potential human and economic costs. Malar J. 2014;13:452.

11. Zhu L, Tripathi J, Rocamora FM, Miotto O, van der Pluijm R, Voss TS, et al. The origins of malaria artemisinin resistance defined by a genetic and transcriptomic background. Nat Commun. 2018;9(1):5158.

12. Bejon $\mathrm{P}$, Lusingu J, Olotu A, Leach A, Lievens $\mathrm{M}$, Vekemans J, et al. Efficacy of RTS,S/AS01E vaccine against malaria in children 5 to 17 months of age. N Engl J Med. 2008;359(24):2521-2532.

13. Palacpac NM, Arisue N, Tougan T, Ishii KJ, Horii T. Plasmodium falciparum serine repeat antigen 5 (SE36) as a malaria vaccine candidate. Vaccine. 2011;29(35):5837-5845.

14. Perrin LH, Merkli B, Loche M, Chizzolini C, Smart J, Richle R. Antimalarial immunity in Saimiri monkeys. Immunization with surface components of asexual blood stages. J Exp Med. 1984;160(2):441-451.

15. Chulay JD, Lyon JA, Haynes JD, Meierovics AI, Atkinson CT, Aikawa M. Monoclonal antibody characterization of Plasmodium falciparum antigens in immune complexes formed when schizonts rupture in the presence of immune serum. J Immunol. 1987;139(8):2768-2774.

16. Kanodia S, Kumar G, Rizzi L, Pedretti A, Hodder AN, Romeo S, et al. Synthetic peptides derived from the C-terminal $6 \mathrm{kDa}$ region of Plasmodium falciparum SERA5 inhibit the enzyme activity and malaria parasite development. Biochim Biophys Acta. 2014;1840(9):2765-2775.

17. Tanabe K, Arisue N, Palacpac NM, Yagi M, Tougan T, Honma H, et al. Geographic differentiation of polymorphism in the Plasmodium falciparum malaria vaccine candidate gene SERA5. Vaccine. 2012;30(9):1583-1593.

18. Chan JA, Fowkes FJ, Beeson JG. Surface antigens of Plasmodium falciparum-infected erythrocytes as immune targets and malaria vaccine candidates. Cell Mol Life Sci. 2014;71(19):3633-3657.

19. Holder AA. The carboxy-terminus of merozoite surface protein 1: structure, specific antibodies and immunity to malaria. Parasitology. 2009;136(12):1445-1456.

20. Remarque EJ, Faber BW, Kocken CH, Thomas AW. Apical membrane antigen 1: a malaria vaccine candidate in review. Trends Parasitol. 2008;24(2):74-84.

21. Lyon JA, Angov E, Fay MP, Sullivan JS, Girourd AS, Robinson SJ, et al. Protection induced by Plasmodium falciparum MSP1(42) is strain-specific, antigen and adjuvant dependent, and correlates with antibody responses. PLoS One. 2008;3(7):e2830

22. Dutta S, Sullivan JS, Grady KK, Haynes JD, Komisar J, Batchelor $\mathrm{AH}$, et al. High antibody titer against apical membrane antigen- 1 is required to protect against malaria in the Aotus model. PLoS One. 2009;4(12):e8138.

23. Horii T, Shirai H, Jie L, Ishii KJ, Palacpac NQ, Tougan T, et al. Evidences of protection against blood-stage infection of Plasmodium falciparum by the novel protein vaccine SE36. Parasitol Int. 2010;59(3):380-386.

24. Okech BA, Nalunkuma A, Okello D, Pang XL, Suzue K, $\mathrm{Li}$ J, et al. Natural human immunoglobulin G subclass responses to Plasmodium falciparum serine repeat antigen in Uganda. Am J Trop Med Hyg. 2001;65(6):912-917.

25. Okech B, Mujuzi G, Ogwal A, Shirai H, Horii T, Egwang TG. High titers of IgG antibodies against Plasmodium falciparum serine repeat antigen 5 (SERA5) are associated with protection against severe malaria in Ugandan children. Am J Trop Med Hyg. 2006;74(2):191-197. 
26. Pang XL, Mitamura T, Horii T. Antibodies reactive with the N-terminal domain of Plasmodium falciparum serine repeat antigen inhibit cell proliferation by agglutinating merozoites and schizonts. Infect Immun. 1999;67(4):1821-1827.

27. Pang XL, Horii T. Complement-mediated killing of Plasmodium falciparum erythrocytic schizont with antibodies to the recombinant serine repeat antigen (SERA). Vaccine. 1998;16(13):1299-1305.

28. Soe S, Singh S, Camus D, Horii T, Druilhe P. Plasmodium falciparum serine repeat protein, a new target of monocyte-dependent antibody-mediated parasite killing. Infect Immun. 2002;70(12):7182-7184.

29. Palacpac NM, Ntege E, Yeka A, Balikagala B, Suzuki N, Shirai $\mathrm{H}$, et al. Phase $1 \mathrm{~b}$ randomized trial and follow-up study in Uganda of the blood-stage malaria vaccine candidate BK-SE36. PLoS One. 2013;8(5):e64073.

30. Frischknecht F, Matuschewski K. Plasmodium Sporozoite Biology. Cold Spring Harb Perspect Med. 2017;7(5):a025478.

31. Cowman AF, Berry D, Baum J. The cellular and molecular basis for malaria parasite invasion of the human red blood cell. J Cell Biol. 2012;198(6):961-971.

32. Weiss GE, Gilson PR, Taechalertpaisarn T, Tham WH, de Jong NWM, Harvey KL, et al. Revealing the sequence and resulting cellular morphology of receptor-ligand interactions during Plasmodium falciparum invasion of erythrocytes. PLoS Pathog. 2015;11(2):e1004670.

33. Beeson JG, Drew DR, Boyle MJ, Feng G, Fowkes FJ, Richards JS. Merozoite surface proteins in red blood cell invasion, immunity and vaccines against malaria. FEMS Microbiol Rev. 2016;40(3):343-372.

34. Gardner MJ, Hall N, Fung E, White O, Berriman M, Hyman RW, et al. Genome sequence of the human malaria parasite Plasmodium falciparum. Nature. 2002;419(6906):498-511.

35. Aoki S, Li J, Itagaki S, Okech BA, Egwang TG, Matsuoka H, et al. Serine repeat antigen (SERA5) is predominantly expressed among the SERA multigene family of Plasmodium falciparum, and the acquired antibody titers correlate with serum inhibition of the parasite growth. J Biol Chem. 2002;277(49):47533-47540.

36. Miller SK, Good RT, Drew DR, Delorenzi M, Sanders PR, Hodder AN, et al. A subset of Plasmodium falciparum SERA genes are expressed and appear to play an important role in the erythrocytic cycle. J Biol Chem. 2002;277(49):47524-47532.

37. McCoubrie JE, Miller SK, Sargeant T, Good RT, Hodder AN, Speed TP, et al. Evidence for a common role for the serine-type Plasmodium falciparum serine repeat antigen proteases: implications for vaccine and drug design. Infect Immun. 2007;75(12):5565-5574.

38. Blackman MJ. Malarial proteases and host cell egress: an 'emerging' cascade. Cell Microbiol. 2008;10(10):1925-1934.

39. Yagi M, Bang G, Tougan T, Palacpac NMQ, Arisue N, Aoshi T, et al. Protective epitopes of the Plasmodium falciparum SERA5 malaria vaccine reside in intrinsically unstructured $\mathrm{N}$-terminal repetitive sequences. PLoS One. 2014;9(6):e98460.
40. Crompton PD, Pierce SK, Miller LH. Advances and challenges in malaria vaccine development. J Clin Invest. 2010;120(12):4168-4178.

41. Barr PJ, Inselburg J, Green KM, Kansopon J, Hahm BK, Gibson HL, et al. Immunogenicity of recombinant Plasmodium falciparum SERA proteins in rodents. Mol Biochem Parasitol. 1991;45(1):159-170.

42. Fox BA, Xing-Li P, Suzue K, Horii T, Bzik DJ. Plasmodium falciparum: an epitope within a highly conserved region of the $47-\mathrm{kDa}$ amino-terminal domain of the serine repeat antigen is a target of parasite-inhibitory antibodies. Exp Parasitol. 1997;85(2):121-134.

43. Fox BA, Horii T, Bzik DJ. Plasmodium falciparum: fine-mapping of an epitope of the serine repeat antigen that is a target of parasite-inhibitory antibodies. Exp Parasitol. 2002;101(1):69-72.

44. Bathurst IC, Gibson HL, Kansopon J, Kahm BK, Green KM, Chang SP, et al. An experimental vaccine cocktail for Plasmodium falciparum malaria. Vaccine. 1993;11(4):449-456.

45. Hill DL, Schofield L, Wilson DW. IgG opsonization of merozoites: multiple immune mechanisms for malaria vaccine development. Int J Parasitol. 2017;47(10-11):585-595.

46. Mishra RP, Oviedo-Orta E, Prachi P, Rappuoli R, Bagnoli F. Vaccines and antibiotic resistance. Curr Opin Microbiol. 2012;15(5):596-602.

47. Six A, Bellier B, Thomas-Vaslin V, Klatzmann D. Systems biology in vaccine design. Microb Biotechnol. 2012;5(2):295-304.

48. Sugiyama T, Suzue K, Okamoto M, Inselburg J, Tai K, Horii T. Production of recombinant SERA proteins of Plasmodium falciparum in Escherichia coli by using synthetic genes. Vaccine. 1996;14(11):1069-1076.

49. Takala SL, Smith DL, Thera MA, Coulibaly D, Doumbo OK, Plowe CV. Short report: rare Plasmodium falciparum merozoite surface protein 1 19-kda (msp-1(19)) haplotypes identified in Mali using high-throughput genotyping methods. Am J Trop Med Hyg. 2007;76(5):855-859.

50. Draper SJ, Angov E, Horii T, et al. Recent advances in recombinant protein-based malaria vaccines. Vaccine. 2015;33(52):7433-7443.

51. Agarwal S, Singh MK, Garg S, Chitnis CE, Singh S. $\mathrm{Ca}(2+)$-mediated exocytosis of subtilisin-like protease 1 : a key step in egress of Plasmodium falciparum merozoites. Cell Microbiol. 2013;15(6):910-921.

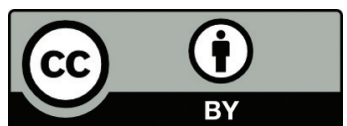

This work is licensed under a Creative Commons Attribution 